\title{
Spectrum of continuous two-contours system
}

\author{
Alexander G. Tatashev ${ }^{1}$ and Marina V. Yashina ${ }^{2}$ \\ ${ }^{1}$ Department of Mathematics, Moscow Automobile and Road Construction State Technical University, 64, Leningradsky prospect, \\ Moscow, Russia \\ ${ }^{2}$ Department of Mathematics, Moscow Automobile and Road Construction State Technical University, 64, Leningradsky prospect, \\ Moscow, Russia
}

\begin{abstract}
A deterministic continuous dynamical system is considered. This system contains two contours. The length of the $i$ th contour equals $c_{i}, i=1,2$. There is a moving segment (cluster) on each contour. The length of the cluster, located on the ith contour, equals $l_{i}, i=1$, 2. If a cluster moves without delays, then the velocity of the cluster is equal to 1 . There is a common point (node) of the contours. Clusters cannot cross the node simultaneously, and therefore delays of clusters occur. A set of repeating system states is called a spectral cycle. Spectral cycles and values of average velocities of clusters have been found. The system belongs to a class of contour systems. This class of dynamical systems has been introduced and studied by A.P. Buslaev.
\end{abstract}

\section{Introduction}

In works of A.P. Buslaev basic approaches for describing flows on complex networks have been laid down. In accordance with these approaches a model is a dynamical system. The supporter of this system is a system of contours with a network structure. Particles (clusters) move on contours in accordance with some rules. Some limitations are imposed on the system. These limitations allow us to study the system analytically.

In [1] (Nagel, Schreckenberg, 1992), a transport model has been introduced. This model is a cellular automaton. In the model, particles move on an infinite or closed sequence of cells in accordance with given rules. In general case, Nagel-Schreckenberg model and its versions are too complicated for analytic research and were studied by simulation.

Analytic results for a simple version of NagelSchreckenberg model have been obtained in [2] (Belitzky, Ferrary, 2005) (a preprint of this paper has been published in 1999). These results have been obtained under assumption that, at any step, each particle moves onto a cell forward if the cell ahead is vacant and does not move if this cell is occupied. It is noted in [2] that the model is equivalent to the elementary cellular automaton 184 (CA 184) in classification of Wolfram, [3]. Results, similar to results of [2], have been obtained independently in [4] (Blank, 2000). In accordance with results of [3], [4], all particles move after some moment at every time for any initial state if the density of particles (the number of particles divided by the number of cells) is not more than $1 / 2$, then the average velocity of particles (the average number of transitions of a particle per time unit) equals $(1-\rho) / \rho$, where $\rho$ is the density. In [5] (Gray, Griffeath, 2001), analytical results have been obtained for somewhat more general traffic model. In [6] (Kanai, 2008), analytical results have been obtained for a stochastic version of the traffic model. Some generalizations of results of [3]-[5] have been obtained in [7] (Blank, 2010).

A two-dimensional traffic model with a toroidal supporter (BML traffic model) has been introduced in [8] (Biham, Middelton, Levin, 1992). In this model, particle move in accordance with a rule, similar to the rule CA 184 . Conditions of selforganization for BML model has been obtained in [9] (Austin, Benjamini, 2006).

The concept of a contour system has been introduced in [10] (Buslaev contour systems).

In [11] (Buslaev, Fomina, Tatashev, Yashina, 2018) the concept of spectrum of a contour system has been introduced for a deterministic dynamical system with a finite set of states. In such system, a sequence of states repeated periodically from some moment. This sequence of states is called a spectral cycle. The system, considered in [11], is a closed chain of contours. Particles move on each contour in accordance with the rule of the cellular automaton 240 (CA 240). There is one cluster on each contour. The spectrum of the system is a set of spectral cycles and corresponding values of clusters velocities. 
In [12] (Buslaev, Tatashev, Yashina, 2016), [13] (Buslaev, Tatashev, 2017) and [14] (Buslaev, Tatashev, 2018), a discrete two-contours system was considered. In this system, particles move on contours in accordance with the rule of CA 184 or CA 240. In [12], [13], the following generalization was also considered. The supporter of the system contains $\mathrm{N}$ contours. There is one common point of the contours. In [12]-[14] theorems have been proved for different versions of movement rules. In [12] [14], mainly, systems with contours of the same length were considered. For a system, containing contours of different lengths, in [13] conditions of self-organization (system resulting in a state of free movement from any initial state) have been obtained.

Buslaev contour systems were also studied in [13] [24].

In this paper, a pair of contours is studied such that the lengths of the contours are different. There is a moving cluster on each contour. There exists a common point of contours (node). Delays occurs at the node. We have been found spectral cycles and obtained formulas for velocities of clusters.

\section{System description}

We consider a discrete dynamical system containing two contours 1 and 2, figure 1. The length of the contour $i$ is equal to $c_{i}, i=1,2$. There is a moving segment cluster (cluster $i$ ) on the contour $i(i=1,2)$. The contours have a common point (node). At any time, each cluster moves in the direction of movement. The cluster $i$ passes the distance $c_{i}$ per $c_{i}$ time units (the velocity equals 1 ) if there is no delay, $i$ $=1,2$. The length of the cluster $i$ is equal to $0<l_{i}<c_{i}$ $, i=1,2$. The coordinate system $\left[0, c_{i}\right]$ is introduced on the contour $i, i=1,2$. The direction of the coordinate axis is reverse to the direction of movement by modulo $c_{i}, i=1,2$. The coordinate of the node is equal to 0 for both contours. The state of the system is the vector

$$
\left(\alpha_{1}(t), \alpha_{2}(t)\right)
$$

where $\alpha_{\mathrm{i}}(\mathrm{t})$ is the coordinate of the leading point of the cluster $i, i=1,2$. We say that the cluster $i$ covers the node at time $t$ if $c_{i}-l_{i}<\alpha_{i}(\mathrm{t})<1, i=1,2$. We say that the cluster $i$ is at the node at time $t$ if $\alpha_{i}(\mathrm{t})=0, i=$ 1, 2. Admissible states of the system are only the states such that no more than cluster covers the node. If at time $t$, the cluster $i$, is at the node, and the other cluster covers the node, then a delay of the cluster $i$ occurs, $i=1$, 2. If both clusters are at the node, i.e., the system is in the state $(0,0)$, then a conflict occurs. In the case of a conflict, only one cluster moves in accordance with a conflict resolution rule. We suppose that the conflict resolution rule is the following. If a conflict occurs at the initial time $t=0$, or a conflict occurs at time $t=t_{0}$ and there are no delays at the time interval $[0, t)$, then, at time $t=t_{0}$, the cluster 1 moves. Assume that a conflict occurs at the time $t=t_{0}$, i.e., $\alpha_{1}\left(t_{0}\right)=\alpha_{2}\left(t_{0}\right)=0$. Suppose the latest delay in the time interval $\left[0, t_{0}\right)$ occurs at time $t=t_{1}$, and, at time $t_{1}$, the cluster $i_{0}, i_{0}=1$, 2 , does not move. Then, at time $t_{0}$, the same cluster $i_{0}$ does not move and the other cluster moves.

The initial state of the system is given. This state should be admissible.

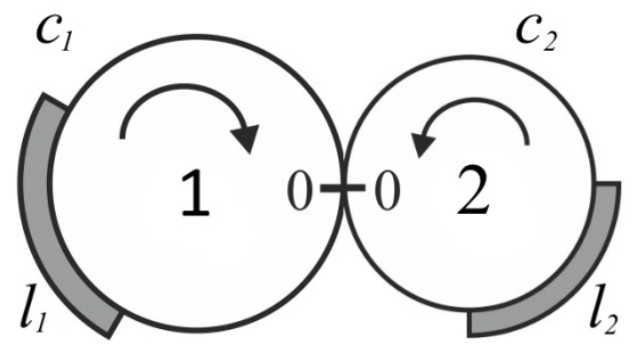

Fig. 1. A two-contours system.

\section{Concepts of spectral cycle, velocity and free movement}

A spectral cycle is a set of states, repeated periodically. Suppose $T$ is the period of the cycle; $H_{i}$ is the distance that the cluster $i$ passes on a spectral cycle for the period), $i=1,2$. The number

$$
v_{i}=\frac{H_{i}}{T}
$$

is called the average velocity of the cluster $i$ on the spectral cycle, $i=1$, 2 .

The system is in state of free movement from some moment $t_{0}$ if both clusters move at any moment $t \geq t_{0}$. If the system results in a state of free movement, then the velocity of clusters is equal to 1 .

\section{Behavior of the system}

We have proved the following theorems, which give conditions of free movement.

Theorem 1. If $\frac{c_{2}}{c_{1}}$ is an irrational number, then the system does not result in a state of free movement from any initial state.

Theorem 2. Suppose $\frac{c_{2}}{c_{1}}$ is a rational number, and $d$ is the greatest common divisor of $c_{1}$ and $c_{2}$, i.e., $d$ is the greatest number such that there exist natural numbers $k_{1}, k_{2}$ satisfying the condition $k_{1} d=c_{1}, k_{2} d$ $=c_{2}$. Then the following is true.

If the condition holds

$$
l_{1}+l_{2} \leq d
$$

then the system results in the state of free movement over a finite time from any initial state (self-organization).

If (1) does not result in the state of free movement over a finite time from no initial state.

Assume that the condition (1) does not hold.

Let us introduce auxiliary parameters $g_{1}, g_{2} b_{1}, b_{2}$ and describe a way to calculate these parameters.

We shall see that, if inequality (1) does not hold, there is one spectral cycle or there are two spectral cycles depending on values of $a_{1}, a_{2}, b_{1}, b_{2}$. The average velocities of clusters depend on these parameters. 
Suppose $A$ is the set of system states such that one cluster does not move in that state; $A_{\mathrm{i}}$ is the set of system states such that the cluster $i$ does not move, $i=1,2$. The set $A_{1}$ contains states $\left(0, \alpha_{2}\right)$, $c_{2}-l_{2}<\alpha_{2}<1$. The set $A_{2}$ contains states $\left(\alpha_{1}, 0\right)$, $c_{1}-l_{1}<\alpha_{1}<1$. We have $A=A_{1} \cup A_{2} \cup(0,0)$. Since the condition of self-organization does not hold, then the system results in the states set $A$ from any initial state over a finite time, and comes out of the set $A$ through the state $\left(0, c_{2}-l_{2}\right)$ or $\left(c_{1}-l_{1}, 0\right)$.

Assume that, at time $t_{0}$, the system is in the state $\left(0, c_{2}-l_{2}\right)$. Let $b$ be a number such that $0<b<l_{2}$. Suppose there exist integer non-negative numbers $k, s$ satisfying the equality

$$
a_{1} k-a_{2} s=c_{2}-l_{2}+b
$$

Due to that (1) does not hold, there exists $k$ and $s$ satisfying the condition.

Let $k_{0}(j)$ be the minimum integer non-negative value of $k$ such that there exists $s$ satisfying the condition (2). Then at time $t_{0}+c_{1} k_{0}(j)$ the system results in the state $\left(0, c_{2}-b\right) \in A_{1}$ if the system has not resulted in another state belonging to $A$.

Denote by $g(b)$ the value $c_{1} k_{0}(b), 0<b<l_{2}$. If for a fixed value 0 there do not exist integer non-negative solutions of (1), then we assume that $g_{1}(b)=\infty$. Let $b$ be an integer number such that $-l_{1}<b<0$. Assume that there exist integer non-negative numbers $k, s$ satisfying (2). Let $k_{0}(j)$ be a minimum integer nonnegative value of $k$ such that there exists $s$ satisfying the condition. Then at time $t_{0}=c_{1} k_{0}(b)+|b|$ the system results in the state $\left(c_{1}-|b|, 0\right) \in A_{2}$ if the system has not resulted in another state belonging to $A$. Denote by $g_{1}(b)$ the value $c_{1} k_{0}(b)+|\mathrm{b}|,-l_{1}<b<0$. If for a given value $b$ there do not exist integer non-negative solutions of (2), we assume that $g_{1}(b)=\infty$. Suppose there are exist integer non-negative numbers $k, s$ such that $c_{1} k-c_{2} S=c_{2}-l_{2}$. Let $k_{0}(0)$ be the minimum integer non-negative number $k$ such that there exist $s$ satisfying the condition. Then at time $t_{0}+k_{0}(b)$ the system results in the state $(0,0)$ if the system has not resulted in another state belonging to $A$. Denote by $g_{1}(0)$ the value $c_{1} k_{0}(0)$. If for $b=0$ there do not exist integer non-negative solutions of (2), then we assume that $g_{1}(0)=\infty$.

Suppose $g_{1}=g_{2}\left(b_{1}\right)=\min g_{1}(b),-l_{1}<b<l_{2}$; then, at time $t_{0}+g_{1}$, the system results either in the state $\left(0, c_{2}-b_{1}\right)$ if $0<b_{1}<l_{2}$, or in the state $\left(c_{1}-\left|b_{1}\right|, 0\right)$ if $-l_{1}<b_{1} \leq 0$, or in the state $(0,0)$ if $b_{1}=0$.

Assume that at time $t_{0}$, the system is in the state $\left(c_{1}-l_{1}, 0\right)$. Let $b$ be an integer number such that $0<b<l_{1}$. Assume that there are exist integer nonnegative numbers $k$, s such that

$$
c_{2} s-c_{1} s=c_{1}-l_{1}+b .
$$

Suppose $s_{0}(j)$ is the minimum integer non-negative number $s$ such there exists integer non-negative number $k$ satisfying the condition (3). Then, at time $t_{0}$ $+s_{0}(j)$, the system results in the state $\left(c_{1}-b, 0\right) \in A_{2}$ if the system has not resulted in another state belonging to the set $A$. Denote by $g_{2}(b)$ the value $c_{2} s_{0}(j), 0<b<l_{1}$. If, for given $b$, there are no integer non-negative numbers satisfying (3), then we suppose $g_{1}(b)=\infty$. Let $b$ be an integer number such that $-l_{2}<$ $b<0$. Assume that there are integer non-negative numbers $k$, $s$ satisfying (3). Let $s_{0}(j)$ be the minimum integer $s$ such that there exists $k$ satisfying the condition. Then at time $t_{0}+c_{2} s_{0}(b)+|b|$ the system results in the state $\left(0, c_{2}-|b|\right) \in A_{1}$ if the system has not resulted in another state belonging the set $A$. Denote by $g_{2}$ (b) the value $c_{2} s_{0}(j)+|j|,-l_{2}<j<0$. If, for given $b$, there are no integer non-negative numbers $k$, $s$ satisfying (4), then we assume that $g_{1}(0)$ $=\infty$. Suppose that there exist integers non-negative numbers such that $c_{2} s-c_{1} k=c_{1}-l_{1}$, and $s_{0}(0)$ is the minimum value of $s$ such that there exists $k$ satisfying the condition. Then the system, at time $t_{0}=c_{2} s_{0}(0)$, results in the state $(0,0)$ if the system has not resulted in another state belonging to the set $A$. If, for $b=0$, there exist no integer non-negative numbers satisfying (3), then we assume that $g_{1}(0)=\infty$.

Denote by $g_{2}(0)$ the value $c_{2} b_{0}(0)$. Suppose $g_{2}=g_{2}(b)=\min (b),-l_{2}<j<l_{1}$. Then, at time $t_{0}+g_{2}$, the system results either in the state $\left(c_{1}-b_{2}, 0\right)$ if $0<b_{2}<l_{1}$, or in the state $\left(0, c_{2}-b_{2}\right)$ if $-l_{2}<b_{2}<0$, or in the state $(0,0)$ if $b_{2}=0$.

Theorem 3. Suppose inequalities $b_{1} \geq 0, b_{2}<0$ hold; then there exist a unique spectral cycle, and this cycle contains the state $\left(0, c_{2}-l_{2}\right)$. The period of the cycle is equal to $g_{1}+l_{2}-b_{1}$. Average velocities of clusters are equal to

$$
v_{1}=\frac{g_{1}}{g_{1}+l_{1}-b_{1}}, v_{2}=1
$$

Proof. Since (2) does not hold, the system does not result in the state of free movement. Hence the system results in a state, belonging the set $A_{1}$, over a finite time, and, after this, in the state $\left(0, c_{2}-l_{2}\right)$, or a state, belonging the set $A_{2}$, and, after this, in the state $\left(c_{1}-l_{1}, 0\right)$. From each of these state, the system result in a state, belonging the set $A_{1} \cup(0,0)$, and, returning in the set $\mathrm{A}$, will be only in the set $A_{1} \cup(0,0)$. On the spectral cycle, the system is in states, not belonging to the set $A_{1}$ (both clusters move in these states), during $g_{1}$ time units and the system is in states, belonging to the set $A_{1}$ (only the cluster 2 moves in these states), during $l_{1}-b_{1}$ time units. From this, Theorem 3 follows.

Theorem 4. Suppose inequalities $b_{1}<0, b_{2} \geq 0$ hold; then there exists a unique spectral cycle, and this cycle contains the state $\left(c_{1}-l_{1}, 0\right)$. The period of this spectral cycle equals $g_{2}+l_{1}-b_{2}$. Velocities of clusters are equal to

$$
v_{1}=1, v_{2}=\frac{g_{2}}{g_{2}+l_{1}-b_{2}},
$$

Theorem 5. Suppose inequalities $b_{1}<0, b_{2}<0$ hold. Then there a unique spectral cycle. The spectral cycle contains the states $\left(c_{1}-l_{1}, 0\right)$ and $\left(0, c_{2}-l_{2}\right)$. The period of the spectral cycle equals $g_{1}+g_{2}+l_{1}+l_{2}-\left|b_{1}\right|-\left|b_{2}\right|$. Velocities of clusters are equal to

$$
\begin{aligned}
& v_{1}=1-\frac{c_{2}-\left|b_{2}\right|}{g_{1}+g_{2}+l_{1}+l_{2}-\left|b_{1}\right|-\left|b_{2}\right|}, \\
& v_{2}=1-\frac{c_{1}-\left|b_{1}\right|}{g_{1}+g_{2}+l_{1}+l_{2}-\left|b_{1}\right|-\left|b_{2}\right|} .
\end{aligned}
$$

Theorems 4,5 are proved similarly to Theorem 3 . 
Theorem 6. Suppose inequalities $b_{1} \geq 0, b_{2} \geq 0$ hold. Then there are two spectral cycles. One of these cycles contains the state $\left(0, c_{2}-l_{2}\right)$. The period of this cycle equals $g_{1}+l_{2}-b_{1}$. On this cycle, velocities of clusters are equal to

$$
v_{1}=\frac{g_{1}}{g_{1}+l_{2}-b_{1}}, v_{2}=1
$$

The other cycle contains the state $\left(c_{1}-l_{1}, 0\right)$. The period of this cycle equals $g_{2}+l_{1}-b_{2}$. On this cycle, velocities of clusters are equal to

$$
v_{1}=1, v_{2}=\frac{g_{2}}{g_{2}+l_{1}-b_{2}} \text {. }
$$

Proof. Depending on the initial state, the system results in a state of the set $A_{1}$ or in the set $A_{2}$. If the system is in the state of the set $A_{1}$, then, returning to the set $A$, the system will only in states of $A_{1} \cup(0,0)$. If the system is in the state of the set $A_{2}$, then, returning to the set $A$, the system will only in states of $A_{2} \cup(0,0)$. The rest of the proof of Theorem 6 is similar to the proof of Theorem 3 .

Example 1. Suppose $c_{1}=4, l_{1}=2, c_{2}=6, l_{2}=2$.

In this case we have

$$
b_{1}=0, g_{1}=4, b_{2}=0, g_{2}=6 \text {. }
$$

There exist two spectral cycles. On one of these cycles, the clusters move with velocities

$$
v_{1}=\frac{2}{3}, v_{2}=1 \text {. }
$$

The period of this cycle equals $T=6$.

On the other spectral cycle, the clusters move with velocities

$$
v_{1}=1, v_{2}=\frac{3}{4} .
$$

The period of this cycle equals $T=8$.

\section{Conclusion}

We study the spectrum of two-contours system with contours of different lengths. The length of the contour $i$ is equal to $c_{i}, i=1,2$. There is a moving cluster on each contour. The length of the cluster, moving on the contour $i$, is equal to $l_{i}, i=1,2$. There is a common point of the contours. This point is called the node. More than one cluster cannot cross the node simultaneously. A cluster stops if it comes to the node at time such that at this time the other cluster crosses the node. If clusters come to the node simultaneously, then only one the cluster moves in accordance with a conflict resolution rule. A set of states such that these states are repeated periodically is called a spectral cycle. We say that the system has the property of self-organization if the system results in the state of free movement over a finite time. In this paper, we have obtained conditions of selforganization. We have proved that, if the condition of self-organization does not hold, then, depending on $c_{1}, c_{2}, l_{1}, l_{2}$, there are one or two spectral cycles. Formulas for average velocities of clusters have been obtained.

\section{Acknowledgements}

This work has been supported by the Russian Foundation for Basic Research, Grant No. 17-0100821-a and Grant No. 17-07-01358-a.

\section{References}

1. K. Nagel, M.A. Schreckenberg. Cellular automation models for freeway traffic $\mathrm{J}$. Phys. I. (DOI: 10.1051/jp1.1992277) 2(12) 2221- 2229 (1992)

2. V. Belitzky, P.A. Ferrary. Invariant measures and convergence properties for cellular automation 184 and related processes J. Stat. Phys. (DOI: 10.1007/s10955-044-8822-4) 118(3) 589623 (2005)

3. S. Wolfram. Statistical mechanics of cellular automata Rev. Mod. Phys. 55 601-644 (1983)

4. M.L. Blank. Exact analysis of dynamical systems arising in models of flow traffic Russian Math Surveys (DOI:.org/10.4213/rm295) 55(5) 562-563 (2005)

5. L. Gray, D. Grefeath The ergodic theory of traffic jams. J. Stat. Phys. (DOI: 10.1023/A:1012202706850) 105 (3/4) 413452 (2001)

6. Kanai M. Exact solution of the zero range process Journal of Physics A. Mathematical and Theoretical (DOI:10.1088/17518118/40/26/001) 40 (19) 7127-7138 (2007)

7. Blank M. Metric properties of discrete time exclusion type processes in continuum. J. Stat. Phys. (DOI: 10.1007/s10955-0109983-y) 140(1) 170-197 (2010)

8. Biham O., Middleton A.A., Levine D. Selforganization and a dynamical transition in traffic-flow models. Phys. Rev. A (DOI: 10.1003/PhysRevA.46.R6124) $\quad$ 46(10) R6124-R6127 (1992)

9. Austin T., Benjamini I. For what number of cars must self-organization occur in the Biham-Middleton-Levine traffic model from any possible starting configuration? arXiv.math/0607759

10. V.V. Kozlov, A.P. Buslaev, A.G. Tatashev On synergy of totally connected flow on chainmails (CMMSE-2013, Cadis, Spain) 3 861-6 873 (2013)

11. A.P. Buslaev, M.Yu. Fomina, A.G. Tatashev, M.V. Yashina. On discrete flow networks model spectra: statement, simulation, hypotheses. J. Phys.: Conf. Ser. (DOI: 10.1088/1742/6596/1053/1/012034) 1053012034 (2018)

12. A.P. Buslaev, A.G. Tatashev, M.V. Yashina. About synergy of flows on flower (DepCoSRELCOMEX 2016, Brunow, Poland) Springer 75-84 (2016) 
13. A.P. Buslaev, A.G. Tatashev. Flows on discrete traffic flower. Journal of Mathematics Research (DOI 10.5539/jmr.v9n1p98) 9(1) 98-108 (2018)

14. A.P. Buslaev, A G. Tatashev. Exact results for discrete dynamical systems on a pair of contours. Math. Meth. Appl. Sci. (DOI:10.1002/mma.4822), February (2018)

15. A.P. Buslaev, A.G. Tatashev, M.V. Yashina. Qualitative properties of dynamical system on toroidal chainmail. (ICNAAM-2013, Rhodes, Greece) AIP Conference Proceedings 1558 1144-1147 (2013)

16. V.V. Kozlov, A.P. Buslaev, A.G. Tatashev. Monotonic walks on a necklace and coloured dynamic vector. Int J Comput Math (DOI 1080/00207160.2014/915964) 92(9) 1910 - 1920 (2015)

17. V.V. Kozlov, A.P. Buslaev, A.G. Tatashev. A dynamical communication system on a network J Comput Appl Math (DOI 10.1016/j.cam.2014.07.026) $275 \quad 247-261$ (2015)

18. V.V. Kozlov, A.P. Buslaev, A. G. Tatashev and M.V. Yashina. Dynamical systems on honeycombs. (Traffic and Granular Flow '13. Springer Verlag, Heidelberg 441452 (2015)

19. V.V. Kozlov, A.P. Buslaev, A G, Tatashev. On real-valued oscillations of a bipendulum. Appl Math Lett (DOI 10.1016/j.aml.2015.02.003) $46 \quad 44-49$ (2015)

20. A.P. Buslaev , A.G. Tatashev, M.V. Yashina. On irrational oscillations of a bipendulum (DepCoS-RELCOMEX, Brunow, Poland) Springer 365 57-63 (2015)

21. A.P. Buslaev, M.V. Yashina. On holonomic mathematical F-bipendulum. Math. Meth. App. Sci., 39, 4820-4828(2016)

22. A. P. Buslaev, A.G. Tatashev, M.V. Yashina. Flows spectrum on closed trio of contours Eur. J. Pure Appl. Math. (DOI 10.29020/nybg.ejpam.v11i1.3201) 11(3) 893-897 (2018).

23. A.P. Buslaev, A.G.Tatashev, M.V. Yashina. On cellular automata, traffic, and dynamical systems in graphs. Int. J. Eng. Technol. (DOI: 10.114419/ijet.v7i2.28.13210) 7(2.28) 351-356 (2018)

24. A.P. Buslaev, A.G. Tatashev. Spectra of local cluster flows on open chain of contours.Eur.J.PureAppl.Math.(DOI10.2902 0/ny/by.ejpam.v.Mi3.3292) 11(3) 628-641 (2018) 